\title{
Management of threadworm infestation during pregnancy
}

Parasitic worms commonly engender feelings of digust in the eye of the beholder', and when the beholder is pregnant, such feelings are likely to be particularly acute. Threadworm infestation (enterobiasis), unless overwhelming, is not usually considered a serious threat to health, although it has been blamed for a wide variety of maladies. These considerations aside, the condition is aesthetically unpleasant and can lead to acute anal irritation or pain that may interfere with sleep and prove socially embarrassing. Experience gained in a drug advisory service suggests that the problem of threadworm infestation during pregnancy is frequently encountered in medical practice and it is hoped that the following brief overview will provide some help to the prescriber.

The true incidence of threadworm (Enterobius vermicularis) infestation among the adult population is unknown but reputed improvements in the social conditions of the population do not appear to have significantly impeded threadworm survival. Its life cycle is well described (albeit not completely unravelled) and a detailed account would be inappropriate for this article. Suffice it to comment that the threadworm (during its nocturnal excursions) deposits very large numbers of eggs upon the perianal skin causing the host intense irritation; the latter effect is partly attributable to the adhesive used for egg attachment. The act of scratching traps ova beneath the finger nails and, if personal hygiene is wanting, reinfestation occurs by oral ingestion. The duration of this cycle is approximately four to six weeks.

Faced with the pregnant patient, who is simultaneously infested with threadworms, the choice of drug is rendered difficult by a relative lack of data combined with overtones of fetal hazard.

\section{Piperazine}

Piperazine (Antepar, Wellcome; Pripsen, Reckitt and Colman) is readily absorbed from the gastrointestinal tract and in the case of Ascaris lumbricoides is known to block the stimulant action of acetylcholine at the myoneural junction. Succinate production is also inhibited. Curiously its mode of action against threadworms has not yet been established. Although piperazine has a long history of use reports of associated teratogenicity are sparse (personal communication, Reckitt and Colman), ${ }^{1}$ although details of two possibly relevant cases have been reported to Reckitt and Colman. In one instance a baby with bilateral hare lip, cleft palate, and anophthalmia, was born to a mother who had taken Pripsen at 12 and 14 weeks' gestation. The remaining incident involved an infant with an abnormality of the right foot whose mother had taken Pripsen at six and eight weeks of pregnancy. These reports have led to a revision of data sheet recommendations, current advice being to avoid use during the first trimester and preferably to delay treatment until after parturition. A further, perhaps theoretical consideration, is that piperazine is potentially neurotoxic, although this is unlikely to be a problem at normal dosage in patients with adequate renal function. Any effects upon the fetal neural system remain a matter of speculation and have not, to date, been demonstrated clinically. In view of the fact that piperazine is excreted in breast milk, the manufacturers advise that the infant recipient should be fed immediately before maternal dosing; breast feeding should then be avoided for the next eight hours during which the milk should be expressed and discarded.

\section{Mebendazole}

Mebendazole (Vermox, Janssen) is, in contrast to piperazine, poorly absorbed from the gastrointestinal tract. Although its exact mode of action in susceptible helminths (of which there are many) remains unknown, it appears to induce selective and irreversible inhibition of glucose uptake thus producing 'diabetic' worms. Its poor gastrointestinal absorption should provide fetal protection. Studies in the rat, however, ${ }^{2}$ have revealed both teratogenic and embryotoxic tendencies on the part of mebendazole (as well as an ability to induce kinky tails in mice offspring ${ }^{3}$ ). The manufacturers of the drug have received several reports of dysmorphogenic effects associated with its use in human pregnancy, although of a range and rate of incidence which are comparable with control values (personal communication). This evidence has prompted warnings against its use during gestation. Evidence that mebendazole can inhibit lactation is based on a single case report and it is unlikely that it reaches breast milk in significant amounts.

\section{Pyrantel}

Pyrantel (Combantrin, Pfizer) is a depolarising neuromuscular blocking agent that acts by causing the release of acetylcholine and inhibiting cholinesterase. It has a long history of use in developing countries but there is little if any evidence regarding its use during pregnancy or lactation. Despite one report suggesting that pyrantel can be used safely in pregnancy, ${ }^{4}$ and evidence that it is poorly excreted in breast milk, ${ }^{5}$ the lack of data dictates caution. Like mebendazole, pyrantel is poorly absorbed from the gastrointestinal tract and has a wide safety margin.

\section{Thiabendazole}

Thiabendazole (Mintezol, Merck Sharp and Dohme) has an extremely wide range of anthelmintic action and is therefore a favourite for treating mixed worm infestation; it is readily absorbed from the gastrointestinal tract. There is animal evidence that it is embryotoxic, ${ }^{67}$ and a study of its use in late human pregnancy has shown a higher incidence of nausea, vomiting, and giddiness than in non-pregnant controls. ${ }^{8}$ Altogether $90 \%$ of an ingested dose of thiabendazole is excreted within 48 hours and this would be a reasonably safe interval to wait before the resumption of breast feeding after taking a dose.

Bearing in mind the obvious limitations of the available anthelmintics, the counsel of perfection in dealing with the pregnant threadworm sufferer is to attempt a rigid regimen of personal hygiene. This measure should, if the facts of threadworm life are to be believed, lead to 'natural' eradication. The mother to be should be asked to vigorously scrub both hands and nails after each bowel evacuation and to take daily baths with particular attention to perianal hygiene. Pyjamas rather than a nightdress are recommended and night attire and clothes should be washed daily. At the same 
time, other members of the family who may be reservoirs of infection, should receive appropriate drug treatment. The opportunity might be taken to explain the grounds for reservation concerning systemic treatment. If immediate eradication is considered important and/or 'natural' eradication is problematic, piperazine would be a reasonable choice, delaying wherever possible to at least the end of the first trimester. Should perianal irritation cause lack of sleep, judicious use of a soothing cream may be helpful.

North West Regional Drug

F N LEACH

Information Service,

St Mary's Hospital,

Whitworth Park,

Manchester M13 OfH
The invaluable assistance of the staff of the medical departments of Janssen Pharmaceutical Ltd Merck the staff of the medical deparpents on and the Pharmaceutical Ltd, Merck Sharp and Dohme Ltd, and Pfizer Ltd and the
Wellcome Medical Division is gratefully acknowledged. The advice of the Trent Wellcome Medical Division is gratefully acknowledged. The advice of the Trent
Regional Drug Information Service was kindly offered during the course of preparation of this paper.

1 Meyer HH, Brenner P. Cleft hand and cleft foot abnormalities as a possible teratogenic side effect of the anthelmintic piperazine. Intermist (Berlin) 1988; 29:217-9.

2 Keystone JS, Murdoch JK. Mebendazole. Ann Intern Med 1979;91:582-8. 3 Baskerville $M$, Wood $M$, Newton CM. Mebendazole for worming mice; effectiveness and side effects. Laboratory Animals 1988;22:263-8.

4 Jean-Pastor MJ. Anti-infective treatment in pregnant women. Mediterranee Medicale 1979;7:55-60.

$5 \mathrm{Katz} M$. Anthelmintics - currents concepts in the treatment of helminthic conditions. Drugs 1986;32:358-71.

6 Tsuchiya T, Tanaka A, Fukuota M, et al. Metabolism of thiabendazole and teratogenic potential of its metabolites in pregnant mice. Chem Pharm Bull (Tokyo) 1987;35:2985-93.

7 Tschuya T, Tanaka A. Effects of thiabendazole on the mouse limb-bud organ end cell in culture. Toxicol Lett 1986; 30:19-26.

8 Chari MV, Hiremath RS. Thiabendazole (a new broadspectrum anthelmintic) in intestinal helminthiasis. I Assoc Physicians India 1967;15:93-6. 